\title{
Conductive Hearing Loss with Present Acoustic Reflex: A Case Report
}

\author{
Takashi Sakamoto*, Shu Kikuta, Shinji Urata, Kazuo Yasuhara, Yayoi S. Kikkawa, \\ Tatsuya Yamasoba \\ Department of Otolaryngology and Head and Neck Surgery, Graduate School of Medicine, Faculty of Medicine, \\ University of Tokyo, Tokyo, Japan \\ Email: "tsakamoto-tky@umin.ac.jp
}

Received 25 July 2014; revised 25 August 2014; accepted 16 September 2014

Copyright $@ 2014$ by authors and Scientific Research Publishing Inc.

This work is licensed under the Creative Commons Attribution International License (CC BY).

http://creativecommons.org/licenses/by/4.0/

(c) (1) Open Access

\begin{abstract}
Acoustic reflex is a sensitive indicator of middle ear lesions when the tympanic membrane is intact and tympanometry is normal. Acoustic reflex is not usually observed in conductive hearing loss caused by disruption of the ossicular chain including ossicular discontinuity and fixation. Ossicular disruption can show at least partially intact acoustic reflex under a certain condition. Moreover, ossicular discontinuity with a nearly intact acoustic reflex is quite rare and there have been few reports published to date. We here present a rare case of conductive hearing loss with a nearly intact acoustic reflex, and the patient was surgically confirmed to have ossicular discontinuity.
\end{abstract}

\section{Keywords}

\section{Acoustic Reflex, Conductive Hearing Loss, Ossicular Discontinuity}

\section{Introduction}

An air-bone gap (ABG) in pure-tone audiometry (PTA) within the normal bone conduction threshold typically represents disruption of the ossicular chain including ossicular discontinuity and fixation, when the tymapanictympanic membrane (TM) is intact and tympanometry is normal. Acoustic reflex (AR) is a sensitive indicator of middle ear lesions, and is not usually observed in such cases of conductive hearing loss. At least partially present AR is occasionally observed in certain types of ossicular disruption. However, there have been a few reports about present AR in an ossicular discontinuity in the English literature [1]-[3]. Moreover, nearly intact AR in ossicular discontinuity is quite rare. We here report a rare case of nearly intact AR, and the patient was surgically confirmed as having ossicular discontinuity.

\footnotetext{
*Corresponding author.
}

How to cite this paper: Sakamoto, T., Kikuta, S., Urata, S., Yasuhara, K., Kikkawa, Y.S. and Yamasoba, T. (2014) Conductive Hearing Loss with Present Acoustic Reflex: A Case Report. Open Journal of Clinical Diagnostics, 4, 177-181. 


\section{Case Report}

A 41-year-old woman was referred to the Hospital of the University of Tokyo with a chief complaint of hearing loss of the left ear. She had complained of hearing loss after undergoing left ear surgery at the age of 2 years. Otoscopic examination revealed normal TMs of both ears. PTA showed left conductive hearing loss with an ABG of 25 - $50 \mathrm{~dB}$ and the Carhart effect at $2 \mathrm{kHz}$, which indicated apparent elevation of the bone conduction threshold (Figure 1). The speech reception threshold was $100 \%$ at $70 \mathrm{~dB}$. Tympanometry showed a peak at 0 daPa within the normal range, which indicated that the tympanogram was classified into type A (Figure 2). AR was evoked by contralateral sound stimulation, and thresholds at $0.5,1,2$, and $4 \mathrm{kHz}$ of the left ear were 65 , 70, 85, and $90 \mathrm{~dB}$, respectively, which were nearly identical to those of the right ear with slight differences within $10 \mathrm{~dB}$ (Figures 3(a)-(d)). High-resolution computed tomography (HRCT) revealed an ambiguous long process of the incus (Figure 4), which suggested a deficit of the long process. Exploratory tympanotomy was performed via a tympanomeatal approach under general anesthesia. Small lateral tympanotomy revealed that the long process of the incus had disappeared, and several tough, fibrous bands connected the head of the stapes with the residual incus were observed. The foot plate of the stapes was not fixed. Therefore, type 3 tympanoplasty was performed using the trimmed residual incus as the columella.

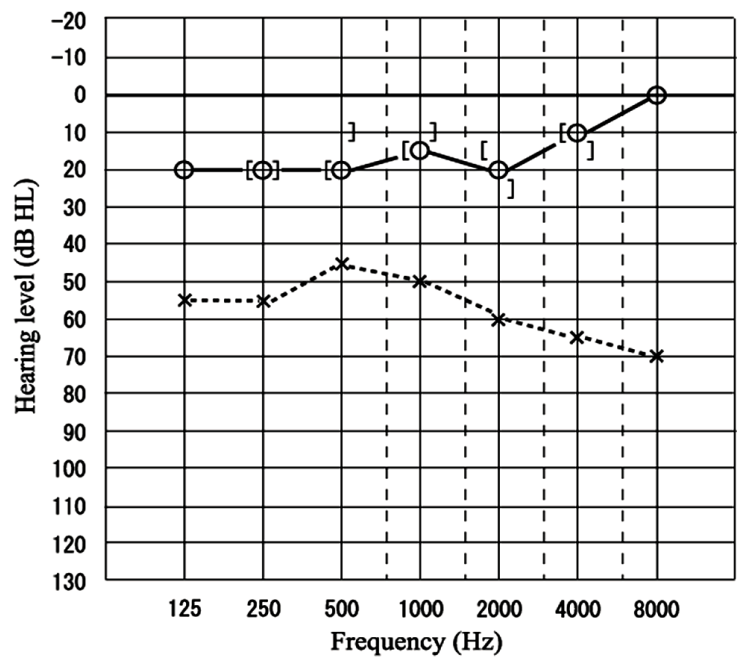

Figure 1. Pure-tone audiometry showed left conductive hearing loss with an ABG of $25-50 \mathrm{~dB}$ and apparent elevation of the bone conduction threshold at $2 \mathrm{kHz}$. The white circles connected by the solid lines and crosses connected by the dashed line show the air conduction thresholds of the right and left ear, respectively. The open bracket, [and closed bracket], indicate the bone conduction thresholds of the right and left ear, respectively.

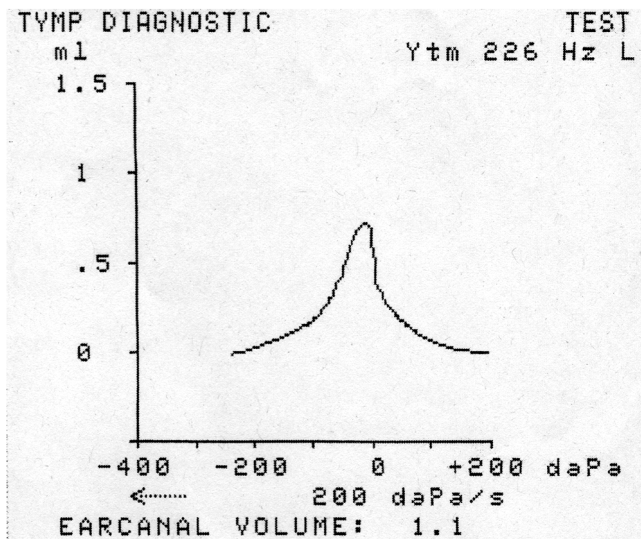

Figure 2. The tympanogram was normal and was classified into type A. 


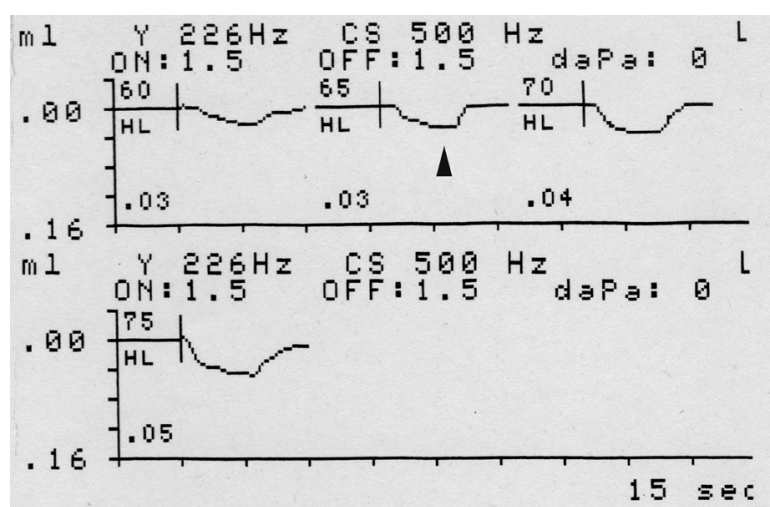

(a)

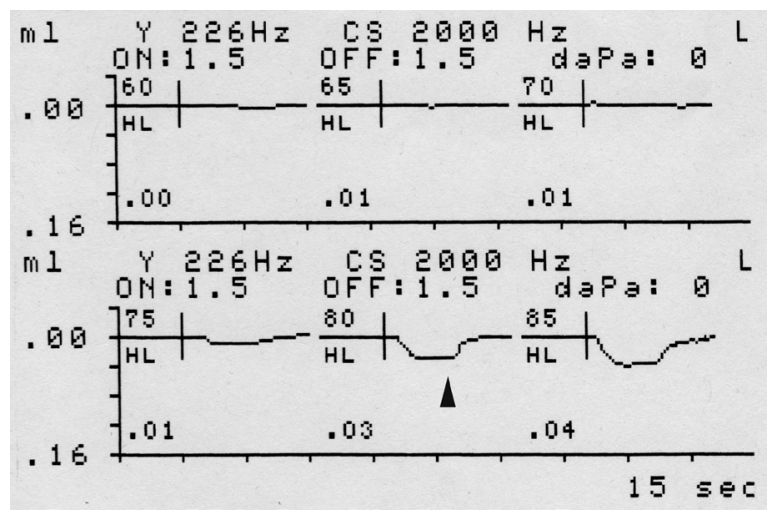

(a)

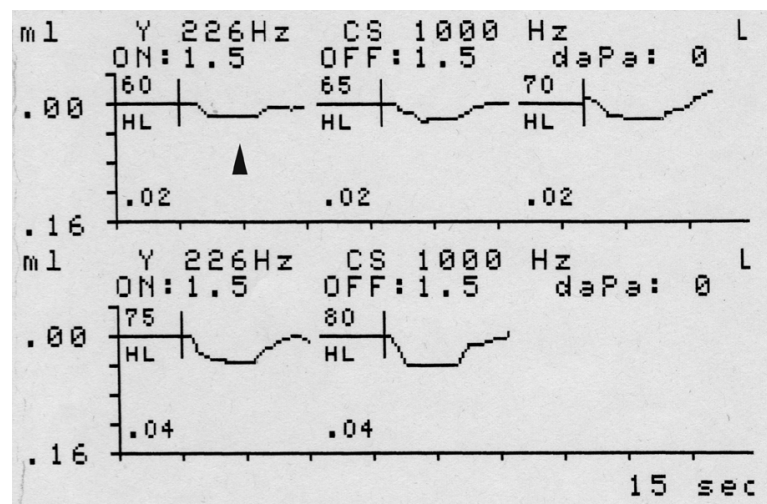

(b)

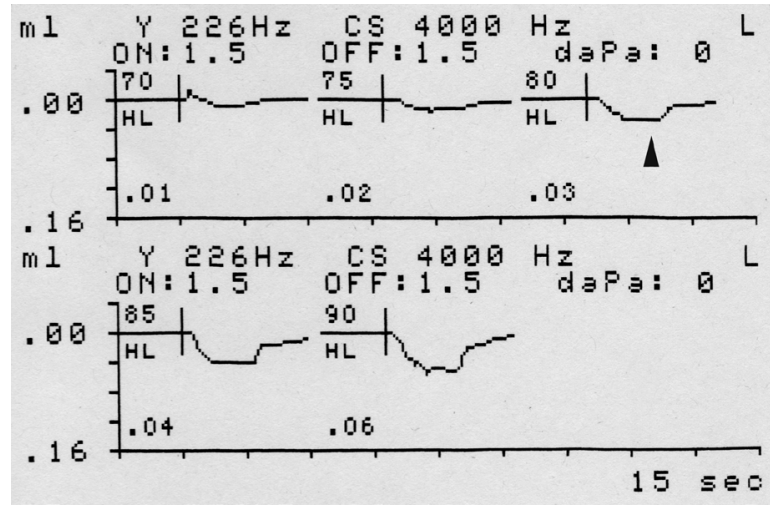

(b)

Figure 3. Acoustic reflex was evoked using contralateral sound stimulation. Wave forms at 0.5 (a); 1 (b); 2 (c); and 4 kHz (d) of the left ear were nearly normal. An arrowhead shows the wave form evoked by the threshold sound pressure at each frequency.

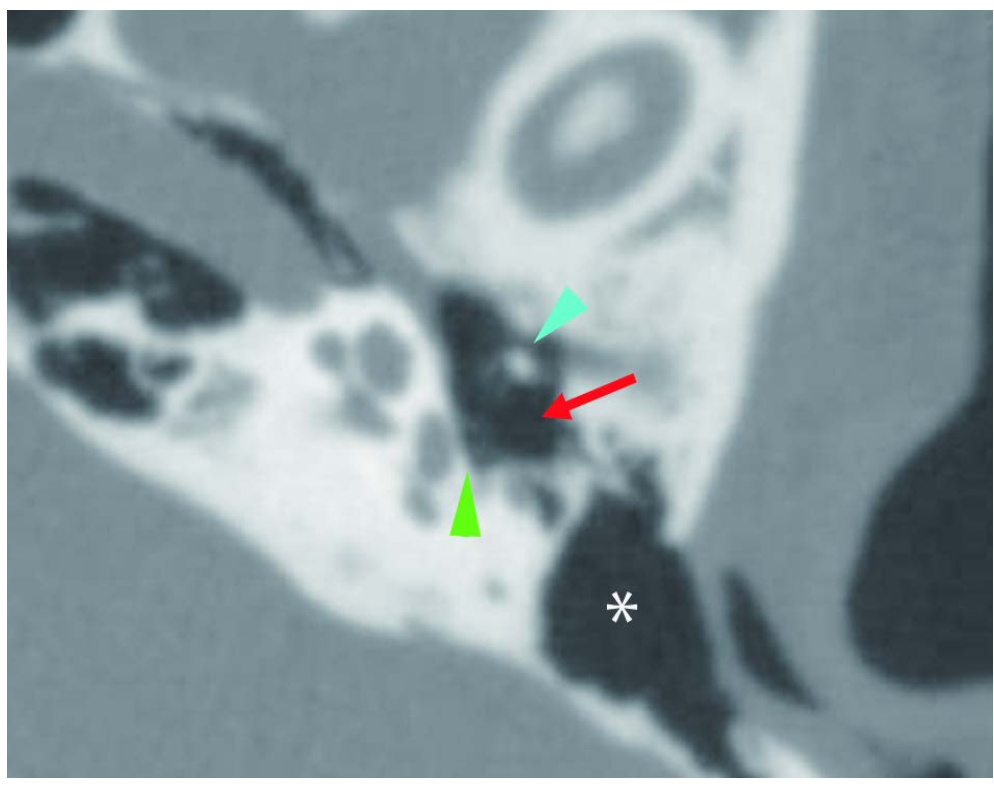

Figure 4. High-resolution computed tomography revealed an ambiguous long process of the incus (red arrow). The handle of the malleus (blue arrow head) and the stapes (green arrow head) were observed. The antrum was opened by a previous surgery (asterisk). 


\section{Discussion}

Herein, we present a rare case of nearly intact AR in spite of ossicular discontinuity.

Conductive hearing loss is defined as an elevated air conduction threshold and a normal bone conduction threshold. Conductive hearing loss with an intact TM is caused by congenital malformations, otosclerosis, ossicular chain fixation, enlarged vestibular aqueduct (EVA), superior semicircular canal dehiscene (SSCD), or an overhanging facial nerve (FN) to the stapes at the tympanic portion [4]. The most common cause of conductive hearing loss is otosclerosis [5], and among all the potential causes listed above, EVA, SSCD, and an overhanging FN to the stapes have been reported to show at least partially intact AR [4] [6] [7]. However, in the present case, HRCT ruled out these causes. The common clinical manifestations for conductive hearing loss caused by ossicular discontinuity are hearing loss and lack of AR. Nevertheless, Oossicular discontinuity can also show at least present AR under certain conditions, including: (1) an intact transmission pathway from the head of the stapes to the TM, (2) replacement of the long process of the incus by a fibrous band, (3) spontaneous myringostapediopexy, and (4) a fibrous union between the stapes and the TM [8] [9]. The present case met the second condition. Nevertheless, it is quite rare that ossicular discontinuity shows nearly intact AR, as observed in the present case, because a fibrous band cannot completely transmit an impedance change to the TM, which is caused by contraction of the stapedius muscle.

AR is the impedance measure of middle ear and its presence implies either normal middle ear function (with normal hearing or a sensorineural hearing loss [SNHL]) or abnormal middle ear function. Therefore, when SNHL is excluded by PTA, AR is a sensitive indicator of middle ear lesions, and an ossicular disruption is strongly suspected when ABG reaches nearly 40 - $50 \mathrm{~dB}$. HRCT can provide additional diagnostic information. In most cases, HRCT can clearly show a defect of the ossicular chain. However, if the ossicles are surrounded by a soft tissue including granulation or a cholesteatoma, it becomes difficult to make an accurate diagnosis of ossicular discontinuity because the contours of the ossicles become ambiguous owing to a partial volume effect. In the present case, HRCT of the axial plane suggested shortening of the long process of the incus. In contrast, the presence of a nearly intact AR suggested that the ossicular chain was preserved. Intraoperative findings revealed that the long process of the incus had disappeared, and that several tough, fibrous bands connected the head of the stapes with the residual incus. Based on these results, we deduced the following reason to explain the presence of the nearly intact AR. The fibrous bands between the head of the stapes and the residual incus were so tough that the impedance change caused by contraction of the stapedius muscle could have been transmitted to the TM without any loss of energy. The present case cautions otologists that presence of ossicular discontinuity cannot be excluded in spite of the presence of AR.

\section{Conclusion}

AR is a sensitive indicator of middle ear lesions and the lack of AR usually indicates a disruption of the ossicular chain, including ossicular discontinuity and fixation. However, the present case showed that nearly intact AR could occur even in the case of ossicular discontinuity under certain conditions. Otologists should be more careful in differential diagnosis of conductive hearing loss based on the AR.

\section{References}

[1] Hayes, D. (1977) Audiologic Quiz Case. Archives of Otolaryngology, 103, 502-503.

[2] Isenberg, S.F. and Tubergen, L.B. (1980) An Unusual Congenital Middle Ear Ossicular Anomaly. Archives of Otolaryngology, 106, 179-181. http://dx.doi.org/10.1001/archotol.1980.00790270043010

[3] Jenkins, H.A., Morgan, D.E. and Miller, R.H. (1980) Intact Acoustic Reflexes in the Presence of Ossicular Disruption. The Laryngoscope, 90, 267-273. http://dx.doi.org/10.1288/00005537-198002000-00011

[4] Ebert Jr., C.S., Zanation, A.M. and Buchman, C.A. (2008) Another Cause for Conductive Hearing Loss with Present Acoustic Reflexes. The Laryngoscope, 118, 2059-2061. http://dx.doi.org/10.1097/MLG.0b013e3181806414

[5] Cox, K.M., Lee, D.J., Carey, J.P. and Minor, L.B. (2003) Dehiscence of Bone Overlying the Superior Semicircular Canal as a Cause of an Air-Bone Gap on Audiometry: A Case Study. American Journal of Audiology, 12, 11-16. http://dx.doi.org/10.1044/1059-0889(2003/004)

[6] Merchant, S.N., Nakajima, H.H., Halpin, C., Nadol Jr., J.B., Lee, D.J., et al. (2007) Clinical Investigation and Mechanism of Air-Bone Gaps in Large Vestibular Aqueduct Syndrome. The Annals of Otology, Rhinology, and Laryngology, 116, 532-541. 
[7] Mikulec, A.A., McKenna, M.J., Ramsey, M.J., Rosowski, J.J., Herrmann, B.S., et al. (2004) Superior Semicircular Canal Dehiscence Presenting as Conductive Hearing Loss without Vertigo. Otology \& Neurotology, 25, 121-129. http://dx.doi.org/10.1097/00129492-200403000-00007

[8] Liden, G., Harford, E. and Hallen, O. (1974) Tympanometry for the Diagnosis of Ossicular Disruption. Archives of Otolaryngology, 99, 23-29. http://dx.doi.org/10.1001/archotol.1974.00780030027005

[9] Zwislocki, J.J. and Feldman, A.S. (1970) Acoustic Impedance of Pathological Ears. ASHA Monographs, 15, 1-42. 
Scientific Research Publishing (SCIRP) is one of the largest Open Access journal publishers. It is currently publishing more than 200 open access, online, peer-reviewed journals covering a wide range of academic disciplines. SCIRP serves the worldwide academic communities and contributes to the progress and application of science with its publication.

Other selected journals from SCIRP are listed as below. Submit your manuscript to us via either submit@scirp.org or Online Submission Portal.
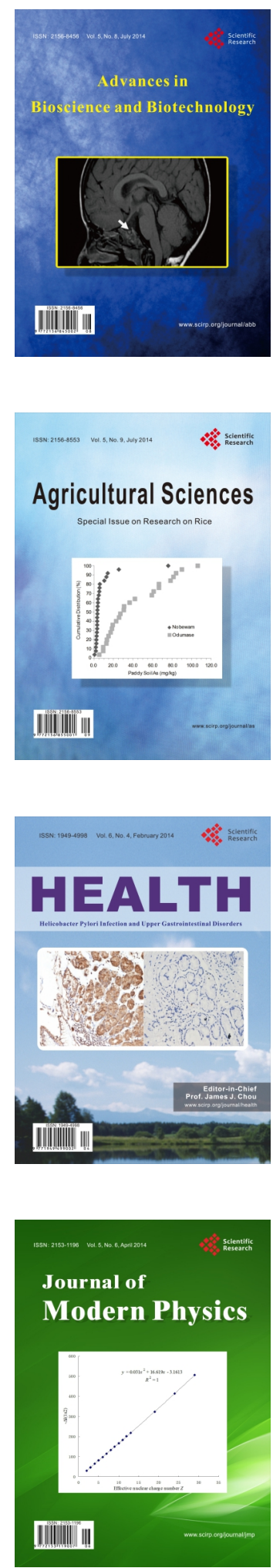
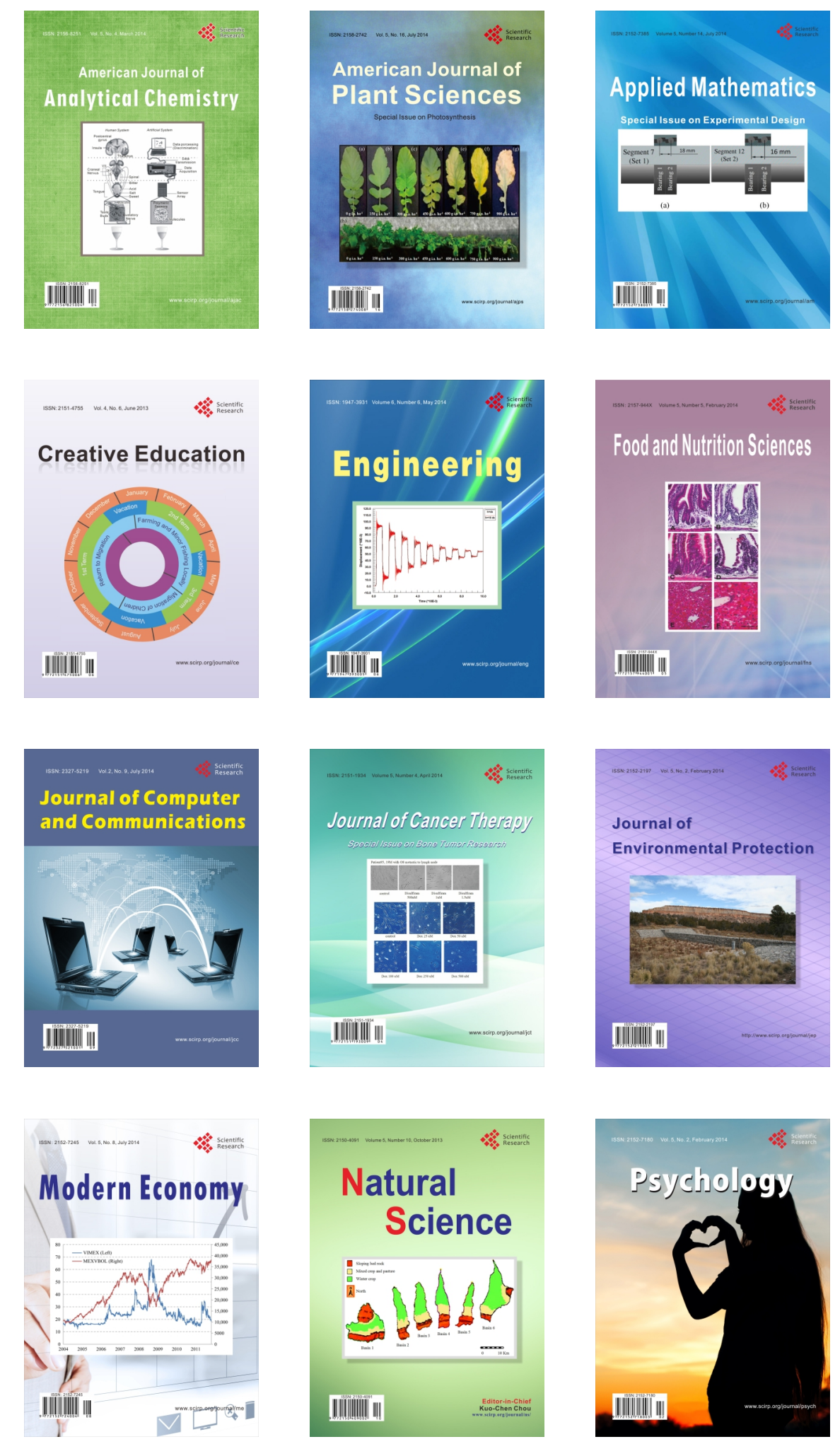